\title{
Particle Size Variation and Prediction of Molecular Weight of Bi(III) Hydrolyzed Polymer Using Light Scattering Technique
}

\author{
N. Priyadarshini, M. Sampath, Shekhar Kumar, and U. Kamachi Mudali \\ Reprocessing R\&D Division, Reprocessing Group, IGCAR, Kalpakkam 603102, India \\ Correspondence should be addressed to Shekhar Kumar; shekhar@igcar.gov.in
}

Received 1 December 2012; Accepted 7 January 2013

Academic Editors: K. Y. Choi and S. Turmanova

Copyright (c) 2013 N. Priyadarshini et al. This is an open access article distributed under the Creative Commons Attribution License, which permits unrestricted use, distribution, and reproduction in any medium, provided the original work is properly cited.

\begin{abstract}
The present paper gives an overview of the hydrolysis reactions up to colloid formation of $\mathrm{Bi}(\mathrm{III})$ in aqueous nitric acid medium using light-scattering measurements. The hydrolysis products of Bi were polynuclear complexes such as dimers, pentamers, and the most important is the hexameric species. In the present investigation $\mathrm{Bi}^{3+}$ polymers were prepared by diluting different concentrations of $\mathrm{Bi}\left(\mathrm{NO}_{3}\right)_{3} \cdot 5 \mathrm{H}_{2} \mathrm{O}$ solutions to $\mathrm{pH} 1 \pm 0.1$ by adding $0.1 \mathrm{M} \mathrm{NaOH}$ solution as it starts to precipitates at $\mathrm{pH}$ 1.4. The degree of polymerization was found to be 5-6 units. Particle size measurements were performed, and it has been found that particle size increases at high concentration of Bi due to aggregation. Refractive index measurements were also performed. The molecular weight of hydrolyzed polymeric species of Bi was determined by using Debye plot, and it was estimated as $1236 \mathrm{Da}$. The second virial coefficient was found to be $6.24 \times 10^{-3} \mathrm{mLg}^{-1} \mathrm{Da}$. The present investigation confirms that the predominant complex in the solution has 5-6 Bi atoms.
\end{abstract}

\section{Introduction}

Among numerous reactions that govern the chemical behavior of metal ions in aqueous medium, the knowledge of hydrolysis, colloid formation, and precipitation is of highest priority. In general, metal ions in aqueous solutions form a variety of hydrolysis complexes, such as mono- and polynuclear hydroxospecies [1]. The polynuclear complexes are formed followed by hydrolysis, and in general it can be represented as

$$
x \mathrm{M}^{n+}+y \mathrm{H}_{2} \mathrm{O} \Longleftrightarrow\left[\mathrm{M}_{x}(\mathrm{OH})_{y}\right]^{(n x-y)}+y \mathrm{H}^{+}
$$

The $\mathrm{pH}$ is the most governing factor where soluble hydrolysis species are present. It is limited due to the formation of colloids or precipitation of hydroxides and oxides.

Aqueous chemistry of $\mathrm{Bi}$ is primarily dominated by trivalent complexes $\left(\mathrm{Bi}^{3+}\right)$ [2]. $\mathrm{Bi}(\mathrm{III})$ readily hydrolyzes in aqueous solutions $\left(\mathrm{p} K_{\mathrm{a}}=1.51\right)$ and has high affinity to oxygen. In addition to that Bismuth is a strong Lewis acid and begins to undergo hydrolysis at low $\mathrm{pH}$ values $(\mathrm{pH} \sim 1)[3,4]$. The $\mathrm{Bi}^{3+}$ aqua ion $\left[\mathrm{Bi}\left(\mathrm{H}_{2} \mathrm{O}\right)_{n}\right]^{3+}$ has been characterized using many techniques which includes extended X-ray absorption fine structure (EXAFS) and low angle X-ray scattering (LAXS) spectroscopy and concluded that 8 water molecules are coordinated to $\mathrm{Bi}^{3+}$. But dynamic simulations show that the number of coordinated water molecules is 9 (i.e., $\left.\left[\mathrm{Bi}\left(\mathrm{H}_{2} \mathrm{O}\right)_{9}\right]^{3+}\right)$ and is found analogous to structures found among lanthanides [5]. Numerous studies on hydrolysis of $\mathrm{Bi}$ show that the predominant species at higher $\mathrm{H}^{+}$concentration is $\mathrm{Bi}^{3+}$. It has been widely agreed that the hydrolyzed species of $\mathrm{Bi}$ are polymeric existing as dimers $\mathrm{Bi}_{2} \mathrm{O}_{2}{ }^{2+}$, pentamers $\mathrm{Bi}_{5} \mathrm{O}_{5}{ }^{5+}$, and the most important among them is the hexameric species $\mathrm{Bi}_{6} \mathrm{O}_{6}{ }^{6+}$ at moderate $\mathrm{Bi}$ concentrations $\left(\geq 10^{-2} \mathrm{~m}\right)$ in acidic solutions with $\mathrm{pH} \sim 2-5$. Further hydrolysis over $\mathrm{pH}$ range of $4-6$ leads to the formation of $\mathrm{Bi}_{9}$ polynuclear complexes [6]. Sedimentation equilibrium measurements indicate that there were 5 or 6 bismuth atoms in the predominant complex present in solutions with approximately 2 hydroxide ions bound per bismuth atom [7]. Since $\mathrm{Bi}-\mathrm{Pb}$ eutectic composition are heavy liquid coolant for nuclear reactors and several recent reactor prototypes use steam-generation modules in direct contact with liquid metal coolant, Bi speciation under hydrothermal conditions is very 
important to optimize the separation of $\mathrm{Bi}$ from concentrates such as $\mathrm{Cu}, \mathrm{Pb}$, and $\mathrm{Mo}[8,9]$. Hence an attempt has been made to study the hydrolysis and polymerization reactions of Bi using light-scattering technique. The use of light scattering to determine the molecular weight of polymers has increased interest because of the several advantages associated with this technique. It provides a nondestructive analytical technique for probing structure and dynamics of polymeric solution. The main aim of this study is to determine the particle size and its changes with change in concentration using dynamic light scattering and weight average molecular weight of $\mathrm{Bi}$ polynuclear complexes by static light scattering.

\section{Theoretical}

2.1. Dynamic Light Scattering. Dynamic light-scattering measurements measure the time dependence of scattered light fluctuations that can be related to the translational diffusion coefficient of the macromolecules. In order to quantify the time dependence, the autocorrelation function $G(\tau)$ is calculated from the product of two photon counts at time $t$ and $t+\tau$ such that $G(\tau)=\langle I(t) I(T+\tau)\rangle[10]$. The normalized intensity autocorrelation function $g(\tau)$ is given by

$$
g^{\text {int }}(\tau)=\frac{\langle I(t) I(t+\tau)\rangle}{\langle I(t) I(t)\rangle}
$$

The intensity autocorrelation function is related to the field autocorrelation function $g^{\text {field }}(\tau)$ by the Siegert relationship

$$
g^{\text {field }}=1+C\left|g^{\text {int }}(\tau)\right|^{2}
$$

where $\tau$ is the delay time and $C$ is the Siegert constant (experimental parameter of the measuring device). For dilute dispersion of macromolecules, $g^{\text {int }}(\tau)$ becomes

$$
g^{\text {int }}(\tau)=e^{-\Gamma_{\tau}}
$$

where $\Gamma$ is the diffusion of particles under investigation. The relation between the diffusion coefficient $(D)$ and the parameter $\Gamma$ can be related by

$$
D=\frac{\Gamma}{q^{2}},
$$

where $q$ is the scattering vector.

From the diffusion coefficient, the hydrodynamic particle radius $R_{h}$ can be derived via the Stokes-Einstein relation

$$
R_{h}=\frac{K_{B} T}{6 \pi \eta D}
$$

where $K_{B}$ is Boltzmann's constant, $T$ is the temperature, and $\eta$ is viscosity of the solvent.

2.2. Static Light Scattering. Static light scattering (SLS) has been widely used to determine the molecular weight of a polymer. SLS measures the time-averaged scattering intensity that can be related to the second virial coefficient and molecular weight by constructing the Debye plot according to the equation

$$
\frac{K C}{R_{\theta}}=\left(\frac{1}{M_{w}}+2 A_{2} C\right) \text {, }
$$

where the optical constant $K=[2 \pi n(d n / d C)]^{2} / N_{A} \lambda_{\circ}^{4}, R_{\theta}$ is the excess Rayleigh ratio, $M_{w}$ is the weight average molecular weight of the solute, $C$ is the concentration (in $\mathrm{g} / \mathrm{mL}$ ), $A_{2}$ is the second virial coefficient, $\lambda_{o}$ is the wavelength of light used, $N_{A}$ is Avogadro's number, and $d n / d C$ is the refractive index increment due to the solute under a given set of solution conditions. A positive value of $A_{2}$ indicates repulsion between molecules, whereas a negative value indicates the presence of intermolecular interactions. The Debye plot is $K C / R_{\theta}$ versus concentration in $\mathrm{g} / \mathrm{mL}$. The intercept of the plot gives $1 / M_{w}$, and the slope is used to calculate the second virial coefficient $[11,12]$.

2.3. Materials and Methods. $\mathrm{Bi}\left(\mathrm{NO}_{3}\right)_{3} \cdot 5 \mathrm{H}_{2} \mathrm{O}$ in crystalline form was obtained from Glaxo laboratories (minimum assay of $98.0 \%$ ). Four different solutions of Bismuth were prepared by dissolving nitrate, $\mathrm{Bi}\left(\mathrm{NO}_{3}\right)_{3} \cdot 5 \mathrm{H}_{2} \mathrm{O}$ in $1 \mathrm{M}$ nitric acid (ARFisher, assay of 69-71\%) to a concentration of $0.0064,0.0121$, 0.0246 , and $0.0367 \mathrm{~g} \mathrm{~mL}^{-1} \cdot \mathrm{Bi}\left(\mathrm{NO}_{3}\right)_{3} \cdot 5 \mathrm{H}_{2} \mathrm{O}$ is of AR grade and used as such. The resulting solutions were diluted by gradually adding $0.1 \mathrm{MNaOH}$ until slight turbidity occurs. The $\mathrm{pH}$ of the solution was maintained as $1 \pm 0.1$. All the solutions were prepared at room temperature. Water used in the experiments was ASTM Grade-I water (from a Millipore Simplicity System with organic removal, 18.2 M. $\Omega . c m$ ). Samples were weighed using Shimadzu (AUW220D) analytical balance with measurement uncertainty of $\pm 0.01 \mathrm{mg}$.

2.4. Measurement of $\mathrm{pH}$ and Refractive Index. The changes in the $\mathrm{pH}$ of the solutions were monitored using Thermo Scientific Orion Star $\mathrm{pH}$ meter. The glass electrode used for the measurement was calibrated with buffer solutions of $\mathrm{pH} 4.01$ and 7.00. The accuracy of $\mathrm{pH}$ measurements was \pm 0.01 units. Refractive index $\left(n_{D}\right)$ of solutions of different concentration of $\mathrm{Bi}$ was measured on Anton Paar RXA 156 refractometer equipped with integrated Peltier thermostat with standard deviation of $2 * 10^{-5} n_{D}$ at $298 \mathrm{~K}$.

2.5. Light-Scattering Measurements. Both SLS and DLS experiments were performed using Microtrac (Nanotrac ULTRA) particle size analyzer. Figures $1(\mathrm{a})$ and 1 (b) represent the experimental light-scattering system used for the present study. The light source was a $780 \mathrm{~nm}, 3 \mathrm{~mW}$ diode laser. An external fiber-optic probe measuring $1.5 \mathrm{~m}$ in length was dipped inside a beaker containing the sample solution. The interface between the sample and the probe is the sapphire window. Initially it reflects the original laser back through the beam splitter to a photodetector. This signal which has the same frequency as the original laser acts as a reference signal, offering heterodyne detection. And then, the laser passes through the sapphire window and is scattered by the particles under Brownian motion. According to the Doppler effect, 


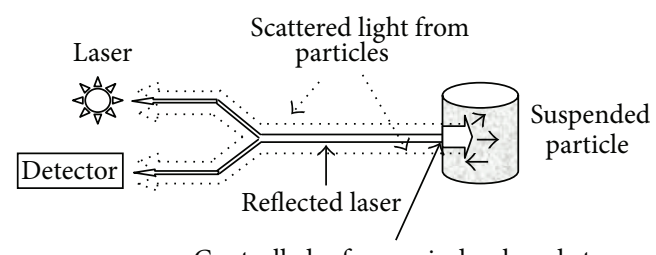

Controlled reference is developed at the interface of the probe tip and fluid

(a)

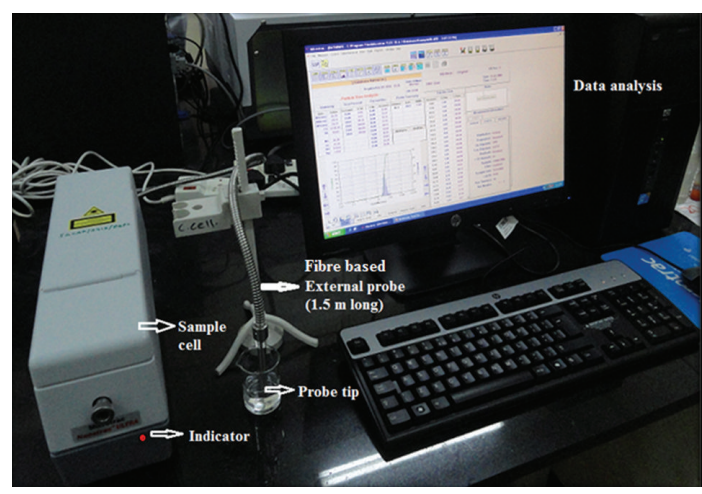

(b)

FIGURE 1: (a) Schematics of the light-scattering system showing the development of controlled reference at the probe tip and fluid interface. (b) Microtrac particle size analyzer with measurement range of $0.8 \mathrm{~nm}$ to $6.4 \mu \mathrm{m}$ used for the present study.

the laser is frequency shifted relative to the velocity of the particle. Light is scattered in all directions including $180^{\circ}$ backwards. This scattered, frequency shifted light is transmitted through the sapphire window to the optical splitter in the probe to the photodetector. These signals of various frequencies combine with the reflected signal of unshifted frequency (controlled reference) to generate a wide spectrum of heterodyne difference frequencies. The power spectrum of the interference signal is calculated with dedicated high speed FFT (Fast Fourier Transform) digital signal processor hardware. The power spectrum is then inverted to give the particle size distribution. The schematic of the instrument is shown in Figure 1. The scattered light was measured at an angle of $180^{\circ}$. Scattering experiments were performed at a temperature of $27 \pm 1^{\circ} \mathrm{C}$. The performance of the instrument was checked with the reference material $(100 \mathrm{~nm}$ polystyrene microsphere suspended in water) prior to particle size measurements. The uncertainty in the measurement was found to be $\pm 1 \mathrm{~nm}$.

\section{Results and Discussion}

Figure 2 shows the comparison plot for varying particle size as the concentration of the solution varies. The $d_{50}$ values range from 121 to $190 \mathrm{~nm}$. The details of each peak are given in Table 1.

The increase in particle diameter is due to aggregation of smaller particles as the concentration increases. The polymer

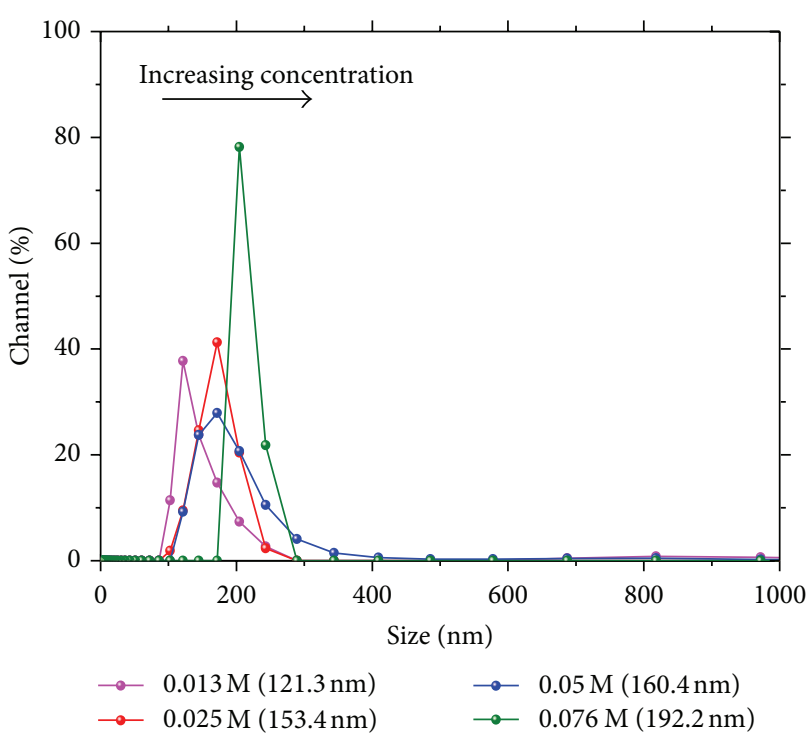

FIGURE 2: A comparison plot for intensity-averaged particle diameter $\left(d_{50}\right)$ variation with different concentration of polymeric Bi solutions prepared in aqueous nitric acid medium.

TABle 1: Peak summary.

\begin{tabular}{lccc}
\hline $\begin{array}{l}\text { Concentration } \\
(\mathrm{g} / \mathrm{mL})\end{array}$ & $\begin{array}{c}\text { Particle diameter } \\
(\mathrm{nm})\end{array}$ & Volume \% & Width \\
\hline 0.0064 & 121.3 & 97.7 & 54.7 \\
0.0121 & 153.4 & 100.0 & 51.8 \\
0.0246 & 160.4 & 100.0 & 81.8 \\
0.0367 & 192.2 & 100.0 & 29.53 \\
\hline
\end{tabular}

TABLE 2: Refractive indices for different concentration of Bi solutions.

\begin{tabular}{lcc}
\hline $\begin{array}{l}\text { Wt. concentration } \\
\left(\mathrm{gmL}^{-1}\right)\end{array}$ & Refractive index & $\begin{array}{c}\text { RI increment } \\
\left(d n / d C \text { in } \mathrm{mL}^{-1}\right)\end{array}$ \\
\hline 0.00634 & 1.33715 & \\
0.01212 & 1.33750 & 0.0842 \\
0.02460 & 1.33866 & \\
\hline
\end{tabular}

is formed as a result of the condensation reaction between the monomeric species. The general equilibrium reaction is given

$$
n \mathrm{Bi}^{3+}+2 n \mathrm{H}_{2} \mathrm{O} \Longleftrightarrow \mathrm{Bi}_{n}(\mathrm{OH})_{2 n}^{n+}+2 n \mathrm{H}^{+}
$$

Figure 3 shows the refractive index increment for different concentrations of $\mathrm{Bi}$, and the values are given in Table 2 . As the concentration increases, the refractive index of the solution also increases giving a positive slope, $d n / d C=$ $0.1304 \mathrm{~mL} \mathrm{~g}^{-1}$. This value has been used as an input for generating the Debye plot. Values of $4.36 \mathrm{gcc}^{-1}$ and 1.9 were assumed as particle density and particle refractive index. Figure 4 shows the Debye plot for different concentration of Bi. It is a straight line with a positive slope. The particles were assumed spherical and ensured that the prepared solution 
TABLE 3: Values of mean diameter, $K C /$ Rop, and hydro-MW for different concentrations of Bi.

\begin{tabular}{lccr}
\hline Weight concentration $(\mathrm{g} / \mathrm{mL})$ & Mean diameter $(\mu \mathrm{m})$ & KC/Rop $(1 / \mathrm{Da})$ & Hydro-MW \\
\hline 0.00640 & 0.4530 & $8.27 E-04$ & $1.28 E+07$ \\
0.01212 & 0.0518 & $8.50 E-04$ & $1.91 E+08$ \\
0.02460 & 0.1262 & $8.85 E-04$ & $2.76 E+09$ \\
\hline
\end{tabular}

TABLE 4: Calculated parameters from the Debye plot.

\begin{tabular}{lcc}
\hline Substance & \multicolumn{2}{c}{ Measured at $\lambda=780 \mathrm{~nm}$} \\
\hline Bi (III) hydrous & $M_{w}(\mathrm{Da})$ & Second virial coefficient, \\
polymer & 1,236 & $A_{2}\left(\mathrm{mLg}^{-1} \mathrm{Da}\right)$ \\
& $6.24 E-03$ \\
\hline
\end{tabular}

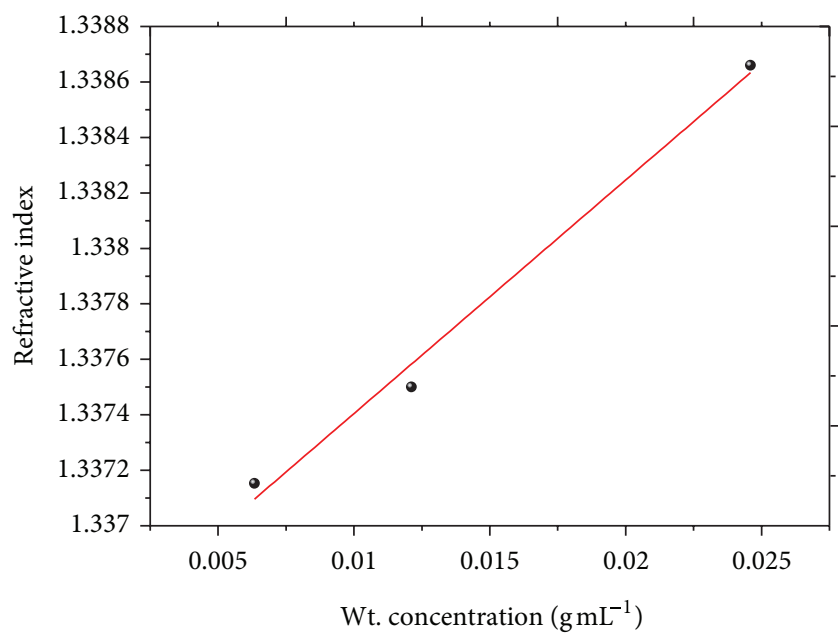

FIGURE 3: The $d n / d C$ curves obtained for different concentration of $\operatorname{Bi}(\mathrm{III})$ solution $\left(R^{2}=0.9827\right)$.

was free of impurities. The molecular weight of the polymer was determined as $1236 \mathrm{Da}$ from the intercept of the plot. Second virial coefficient was determined as $6.24 E-03 \mathrm{mLg}^{-1} \mathrm{Da}$. The positive value of $A_{2}$ shows that the overall net interactive force is repulsive between the solute species and hence stable in the aqueous nitric acid medium. The positive value of the second virial coefficient shows a positive $\Delta G$ for polymer chain interpenetration which leads to steric stabilization. The resulting stabilization could be the result of positive $\Delta H$ or negative $\Delta S$, where positive enthalpy reflects the release of bound solvent and negative entropy reflects the loss of configurational freedom as the polymer chains interpenetrate. At this condition the dispersion is sterically stabilized. Thus nitric acid is found to be a good solvent medium. The values of $K C /$ Rop, mean diameter, and hydro molecular weight of each concentration are given in Table 3 . With increasing concentration of $\mathrm{Bi}$ the degree of polymerization increases. The calculated parameters from the Debye plot are given in Table 4 . The results show that Bi undergoes hydrolysis at low $\mathrm{pH}$ and forms polymers with more than 5 units.

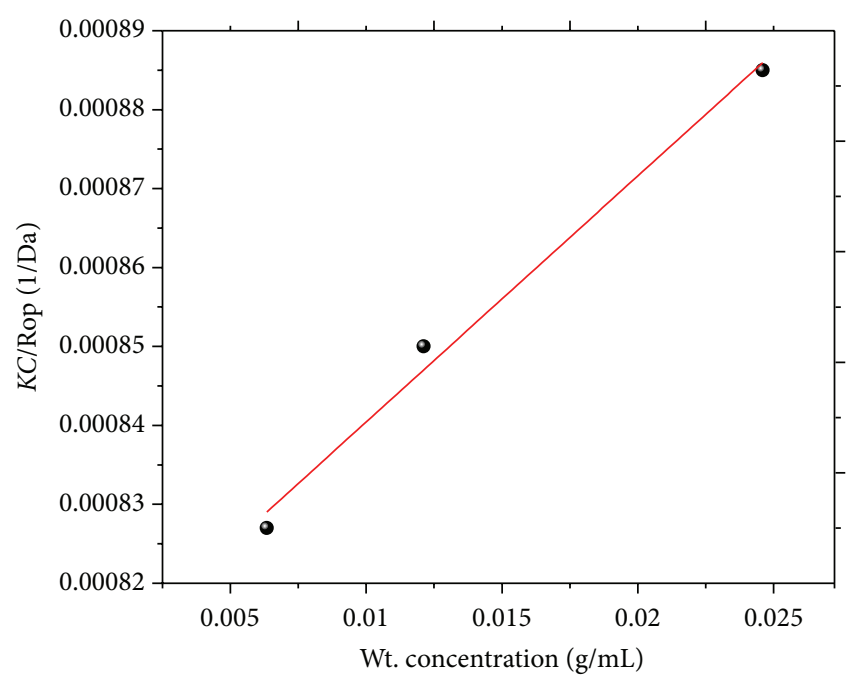

FIGURE 4: Debye plot for various concentrations of $\mathrm{Bi}^{3+}$ with $R^{2}$ of 0.9834 .

\section{Conclusion}

A new approach was applied to interpret the hydrolytic behaviour of $\mathrm{Bi}$ using light-scattering technique. X-ray diffraction, centrifugation, electrophoretic, and tyndallometric studies reported the different species of Bi present in aqueous medium. The present investigation attempts to determine the molecular weight of the hydrolyzed species. It was shown that the particle size increases due to increased aggregation of solute. This shows that polymerization of Bi strongly depends on concentration. The second virial coefficient has also been determined, and the positive value shows that there is repulsion between the molecules making the polymeric solution stable. The weight average molecular weight of polymers of $\mathrm{Bi}(\mathrm{III})$ formed in aqueous nitric acid medium has confirmed that the hydrolyzed species has 5-6 atoms of Bismuth.

\section{References}

[1] N. Torapava, Hydration, solvation and hydrolysis of multicharged metal ions [Doctoral thesis], 2011.

[2] F. A. Cotton, G. Wilkinson, C. A. Murillo, and M. Bochmann, Advanced Inorganic Chemistry, John Wiley \& Sons, New York, NY, USA, 1999.

[3] R. Luckay, I. Cukrowski, J. Mashishi et al., "Synthesis, stability and structure of the complex of bismuth(III) with the nitrogendonor macrocycle 1,4,7,10-tetraazacyclododecane. The role of the lone pair on bismuth(III) and lead(II) in determining coordination geometry," Journal of the Chemical Society-Dalton Transactions, no. 5, pp. 901-908, 1997. 
[4] V. Stavila, R. L. Davidovich, A. Gulea, and K. H. Whirmire, "Bismuth(III) complexes with aminopolycarboxylate and polyaminopolycarboxylate ligands: chemistry and structure," Coordination Chemistry Reviews, vol. 250, no. 21-22, pp. 2782-2810, 2006.

[5] J. Naslund, I. Persson, and M. Sandstrom, "Solvation of the bismuth(III) ion by water, dimethyl sulfoxide, N,N' dimethylpropyleneurea, and N,N-dimethylthioformamide. An EXAFS, large-angle X-ray scattering, and crystallographic structural study," Inorganic Chemistry, vol. 39, no. 18, pp. 4012-4021, 2000.

[6] C. F. Baes and R. E. Mesmer, The Hydrolysis of Cations, John Wiley \& Sons, New York, NY, USA, 1976.

[7] R. S. Tobias and S. Y. Tyree, "Studies on hydrolyzed bismuth(III) solutions. II. Light scattering," Journal of the American Chemical Society, vol. 82, no. 13, pp. 3244-3249, 1960.

[8] D. Gorse-Pomonti and V. Russier, "Liquid metals for nuclear applications," Journal of Non-Crystalline Solids, vol. 353, no. 3240, pp. 3600-3614, 2007.

[9] S. L. Chryssoulis and J. McMullen, "Mineralogical investigation of gold ores," in Developments in Mineral Processing, D. A. Mike and B. A. Wills, Eds., Elsevier, Philadelphia, Pa, USA, 2005.

[10] R. Pecora, Dynamic Light Scattering, Plenum, New York, NY, USA, 1985.

[11] J. Bloustine, V. Berejnov, and S. Fraden, "Measurements of protein-protein interactions by size exclusion chromatography," Biophysical Journal, vol. 85, no. 4, pp. 2619-2623, 2003.

[12] B. J. Berne and R. Pecora, Dynamic Light Scattering with Application in Physics, Chemistry and Biology, John Wiley \& Sons, New York, NY, USA, 1976. 

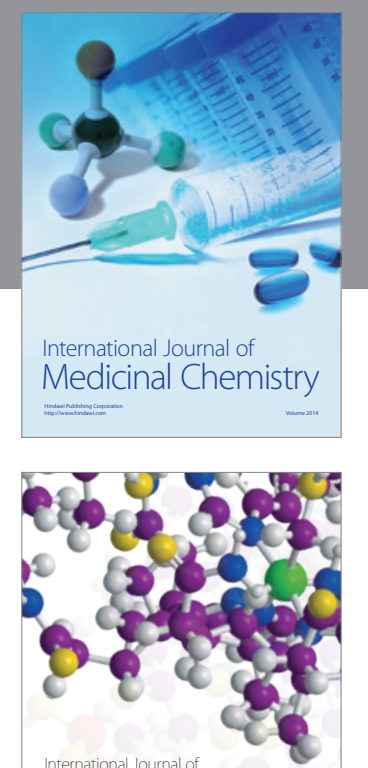

\section{Carbohydrate} Chemistry

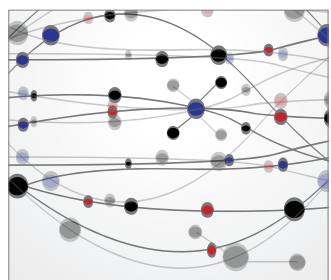

The Scientific World Journal
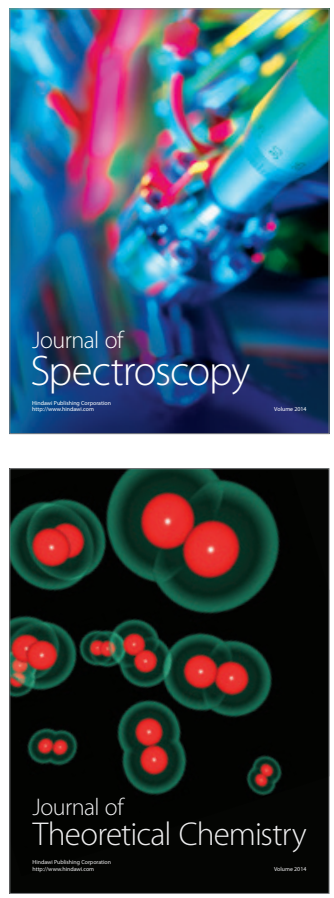
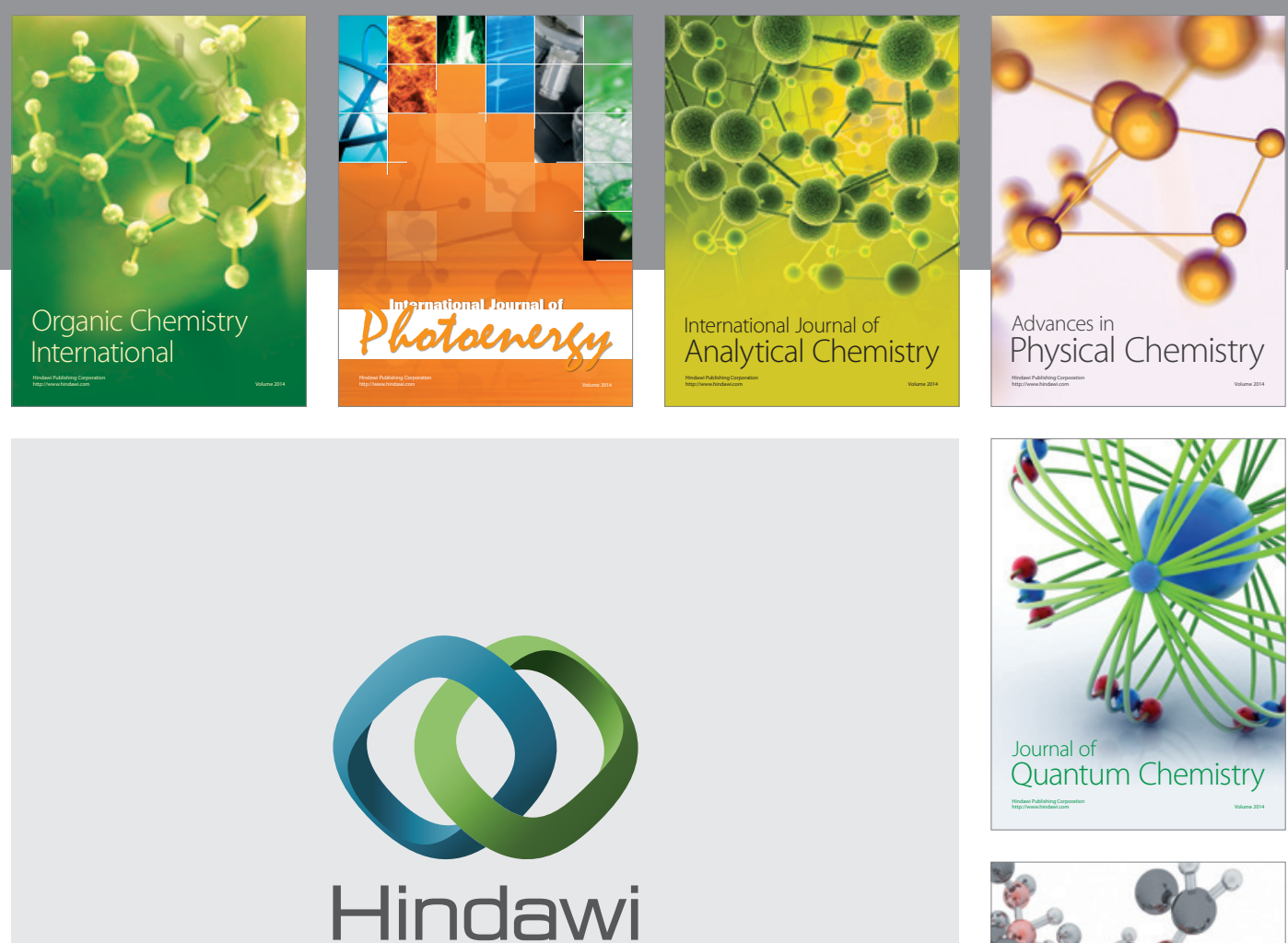

Submit your manuscripts at

http://www.hindawi.com

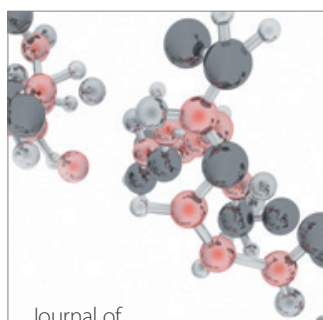

Analytical Methods

in Chemistry

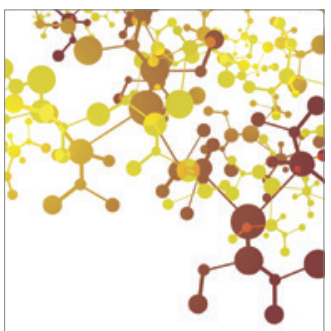

Journal of

Applied Chemistry

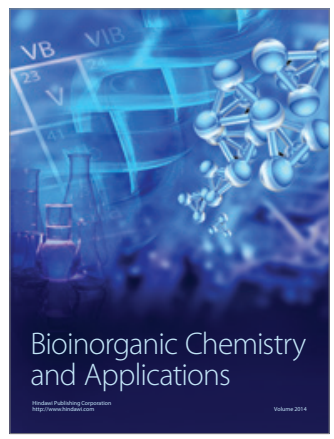

Inorganic Chemistry
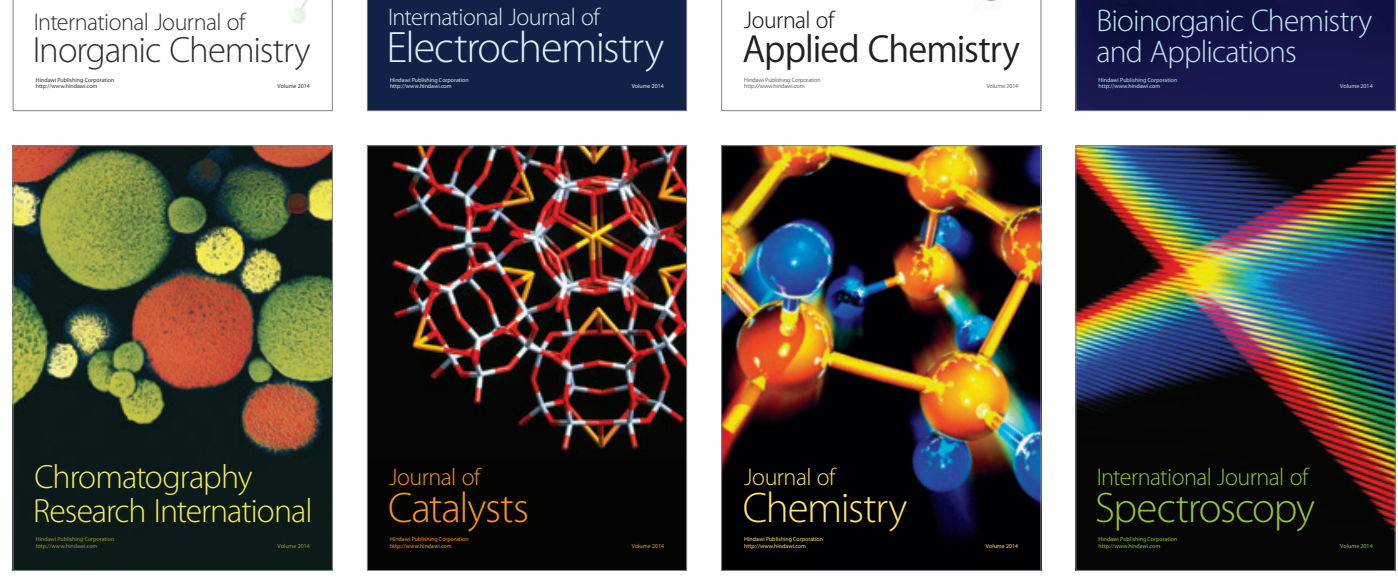